\title{
Singular fractional integro-differential inequalities and applications
}

\author{
Asma Al-Jaser ${ }^{1 *}$ and Khaled M Furati ${ }^{2}$
}

* Correspondence:

asmaljaser@hotmail.com

'Department of Mathematical

Sciences, Princess Nora Bint

Abdulrahman University, Riyadh

84428, Saudi Arabia

Full list of author information is

available at the end of the article

\begin{abstract}
In this article, fractional integro-differential inequalities with singular coefficients have been considered. The bounds obtained for investigating the behavior of the solution of a class of singular nonlinear fractional differential equations has been used, some applications are provided.

2010 Mathematics Subject Classification: 26A33; 34A08; 34A34; 45J05.
\end{abstract}

Keywords: Bihari inequality, fractional differential equations, Riemann-Liouville integral, Cauchy-type problem, singular differential equations

\section{Introduction}

Many physical and chemical phenomena can be modeled with fractional differential equations. However, finding solutions to such equations may not be possible in most cases, particularly the nonlinear ones. Instead, many researchers have been studying the qualitative attributes of the solutions without having them explicitly. In particular, the existence and uniqueness of solutions of a wide class of Cauchy-type problems have been intensively investigated; see for example [1] and the references therein. Also classes of boundary value problems have been considered. For example in $[2,3]$, the authors established the existence and uniqueness of the solution for a class of linear and superlinear fractional differential equations.

Inequalities play an important role in the study of existence, uniqueness, stability, continuous dependence, and perturbation. In [4-7], bounds for solutions of fractional differential inequalities of order $0<\alpha<1$ are obtained. Those bounds are generalizations and extensions of analogous bounds from the integer order case [8,9]. In [5], a number of Bihari-type inequalities for the integer order derivatives are extended to non-integer orders. However, the coefficients of these inequalities are assumed to be continuous at the left end of the interval of definition.

In this article, we extend these inequalities to ones with singular integrable coefficients of the form

$$
\left|D^{\alpha} u(t)\right| \leq a(t)+b(t) \int_{0}^{t} c(s)\left(\sum_{j=0}^{k} \mid D^{\beta_{j}} u(s)\right)^{n} d s,
$$

(c) 2011 Al-Jaser and Furati; licensee Springer. This is an Open Access article distributed under the terms of the Creative Commons Attribution License (http://creativecommons.org/licenses/by/2.0), which permits unrestricted use, distribution, and reproduction in any medium, provided the original work is properly cited. 
and

$$
\left|D^{\alpha} u(t)\right| \leq a(t)+\int_{0}^{t} c(s) \sum_{i=0}^{m}\left|D^{\gamma_{i}} u(s)\right| \sum_{j=0}^{k}\left|D^{\beta_{j}} u(s)\right| d s,
$$

where $0<\alpha<1,0 \leq \beta_{0}<\beta_{1}<\ldots<\beta_{k}<\alpha, 0 \leq \gamma_{0}<\gamma_{1}<\ldots<\gamma_{m}<\alpha, n \geq 1$ is an integer, and $a, b \in C(0, T] \cap L_{1}(0, T)$. Also we give some applications.

The rest of the article is organized as follows. In Section 2, we introduce some definitions and results that we use in our proofs. Section 3 contains the main results. The last section is devoted to some applications.

\section{Preliminaries}

In this section, we introduce some notations, definitions, and lemmas which will be needed later. For more details, we refer the reader to $[1,8,10,11]$.

We denote by $L_{p}, 1 \leq p \leq \infty$, the Lebesgue spaces, and by $A C[a, b]$ the space of all absolutely continuous functions on $[a, b],-\infty<a<b<\infty$.

Definition 1. Let $f \in L_{1}(a, b)$, the integral

$$
I_{a^{+}}^{\alpha} f(x)=\frac{1}{\Gamma(\alpha)} \int_{a}^{x} \frac{f(t)}{(x-t)^{1-\alpha}} d t, x>a, \alpha>0,
$$

is called the Riemann-Liouville fractional integral of order $\alpha$ of the function $f$. Here, $\Gamma(\alpha)$ is the gamma function.

Definition 2. The expression

$$
D_{a^{+}}^{\alpha} f(x)=\frac{1}{\Gamma(1-\alpha)}-\frac{d}{d x} \int_{a}^{x} \frac{f(t)}{(x-t)^{\alpha}} d t, x>a, 0<\alpha<1,
$$

is called the Riemann-Liouville fractional derivative of order $\alpha$ of the function $f$.

Note that $D_{a^{+}}^{\alpha} f(x)=\frac{d}{d x} I_{a^{+}}^{1-\alpha} f(x)$. We use the notation $f_{\alpha}$ to denote $I_{a^{+}}^{\alpha} f$. We set

$$
I_{a^{+}}^{0} f=D_{a^{+}}^{0} f=f .
$$

Definition 3. Let $0<\alpha<1$. A function $f \in L_{1}(a, b)$ is said to have a summable fractional derivative $D_{a^{+}}^{\alpha} f$ on $(a, b)$ if $I_{a^{+}}^{1-\alpha} f \in A C[a, b]$.

Definition 4. We define the space $I_{a^{+}}^{\alpha}\left(L_{p}(a, b)\right), \alpha>0,1 \leq p<\infty$, to be the space of all functions $f$ such that $f=I_{a^{+}}^{\alpha} \varphi$ for some $\phi \in L_{p}(a, b)$.

Theorem 5. A function $f$ is in $I_{a^{+}}^{\alpha}\left(L_{1}\right), 0<\alpha<1$, if and only if $f_{1-\alpha} \in A C[a, b]$, and $f_{1-\alpha}(a)=0$ (see [[11], Theorem 2.3, p. 43]).

Lemma 6. If $\alpha>0$ and $\beta>0$, then

$$
I_{a^{+}}^{\alpha} I_{a^{+}}^{\beta} f(t)=I_{a^{+}}^{\alpha+\beta} f(t),
$$

is satisfied at almost every point $t \in[a, b]$ for $f \in L_{1}(a, b), 1 \leq p \leq \infty$ (see [[1], p. 73]).

Lemma 7. If $f \in A C[a, b]$, then $I^{1-\alpha} f \in A C[a, b], 0<\alpha<1$ (see [[11], Lemma 2.1, p. 33]). 
Corollary 8. If $f \in L_{1}(a, b)$ has a summable fractional derivative $D_{a^{+}}^{\alpha} f, 0<\alpha<1$, on $(a, b)$, then for $0 \leq \beta<\alpha<1$ we have

$$
D_{a^{+}}^{\beta} f(t)=I_{a^{+}}^{\alpha-\beta} D_{a^{+}}^{\alpha} f(t)+\frac{f_{1-\alpha}(a)}{\Gamma(\alpha-\beta)}(t-a)^{\alpha-\beta-1} .
$$

Proof. Since $I_{a^{+}}^{1-\alpha} f \in A C[a, b]$, then we can write

$$
I_{a^{+}}^{1-\alpha} f(t)=I D I_{a^{+}}^{1-\alpha} f(t)+I_{a^{+}}^{1-\alpha} f(a) .
$$

Also from Lemmas 6 and 7 we have

$$
I_{a^{+}}^{1-\beta} f(t)=I_{a^{+}}^{\alpha-\beta} I_{a^{+}}^{1-\alpha} f(t) \in A C[a, b] .
$$

Thus, $f$ has a summable fractional derivative $D_{a^{+}}^{\beta}$ g given by

$$
\begin{aligned}
D_{a^{+}}^{\beta} f(t) & =D I_{a^{+}}^{1-\beta} f(t)=D I_{a^{+}}^{\alpha-\beta}\left(I D I_{a^{+}}^{1-\alpha} f(t)+I_{a^{+}}^{1-\alpha} f(a)\right) \\
& =I_{a^{+}}^{\alpha-\beta} D_{a^{+}}^{\alpha} f(t)+\frac{I_{a^{+}}^{1-\alpha} f(a)}{\Gamma(\alpha-\beta)}(t-a)^{\alpha-\beta-1}
\end{aligned}
$$

Lemma 9. Let $v, f, g$ and $k$ be non-negative continuous functions on $[a, b]$. Let $\omega$ be a continuous, non-negative and non-decreasing function on $[0, \infty)$, with $\omega(0)=0$ and $\omega(u)>0$ for $u>0$, and let $F(t)=\max _{0 \leq s \leq t} f(s)$ and $G(t)=\max _{0 \leq s \leq t} g(s)$. Assume that

$$
v(t) \leq f(t)+g(t) \int_{a}^{t} k(s) \omega(v(s)) d s, t \in[a, b] .
$$

Then

$$
v(t) \leq H^{-1}\left[H(F(t))+G(t) \int_{a}^{t} k(s) d s\right], t \in[a, T),
$$

where $H(v)=\int_{v_{0}}^{v} \frac{d \tau}{\omega(\tau)}, 0<v_{0} \leq v, H^{-1}$ is the inverse of $H$ and $T>a$ is such that $\left[H(F(t))+G(t) \int_{a}^{t} k(s) d s\right] \in \operatorname{Dom}\left(H^{-1}\right)$, for all $t \in[a, T)$ (see [[8], Corollary 5.5]).

Let $I \subset R$, and $g_{1}, g_{2}: I \rightarrow R \backslash\{0\}$ We write $g_{1} \propto g_{2}$ if $g_{2} / g_{1}$ is non-decreasing in $I$.

Lemma 10. Let $f(t)$ be a positive continuous function on $[a, b]$, and $k_{j}(t, s), 1 \leq j \leq n$, be non-negative continuous functions for $a \leq s \leq t<b$ which are monotonic nondecreasing in $t$ for any fixed $s$. Let $g_{j}(u), j=1,2, \ldots, n$, be non-decreasing continuous functions on $[0, \infty)$, with $g_{j}(0)=0, g_{j}(u)>0$ for $u>0$, and $g_{1} \propto g_{2} \propto \ldots \propto g_{n}$ in $(0, \infty)$. If $u(t)$ is a non-negative continuous functions on $[a, b]$ and satisfy the inequality

$$
u(t) \leq f(t)+\sum_{j=1}^{n} \int_{a}^{t} k_{j}(t, s) g_{j}(u(s)) d s, t \in[a, b],
$$


then

$$
u(t) \leq c_{n}(t), a \leq t<T,
$$

where $c_{0}(t)=\max _{0 \leq s \leq t} f(s)$,

$$
\begin{aligned}
& c_{j}(t)=G_{j}^{-1}\left[G_{j}\left(c_{j-1}(t)\right)+\int_{a}^{t} k_{j}(t, s) d s\right], j=1, \cdots, n, \\
& G_{j}(u)=\int_{u_{j}}^{u} \frac{d x}{g_{j}(x)}, u>0, u_{j}>0,
\end{aligned}
$$

and $T$ is chosen so that the function $c_{j}(t), j=1,2, \ldots, n$, are defined for $a \leq t<T$ (see [[8], Theorem 10.3]).

Lemma 11. For non-negative $a_{i}, i=1,2, \ldots, k$,

$$
\left(\sum_{i=1}^{k} a_{i}\right)^{n} \leq k^{n-1} \sum_{i=1}^{k} a_{i}^{n}, n \geq 1
$$

Definition 12. We denote by $C L_{1}(a, b)$ the space of all functions $f$ such that $f \in C(a, b] \cap L_{1}(a, b)$.

Lemma 13. If $f \in C L_{1}(a, b)$, then $I_{a^{+}}^{\alpha} f \in C L_{1}(a, b), \alpha>0$.

Proof. Clearly if $\alpha \geq 1$, then $I_{a^{+}}^{\alpha} f=I_{a^{+}} I_{a^{+}}^{\alpha-1} f \in A C[a, b]$. For $0<\alpha<1$, it follows from Fubini's theorem that $I_{a^{+}}^{\alpha} f \in L_{1}(a, b)$. So, it remains to show that $I_{a^{+}}^{\alpha} f$ is continuous at every $t_{0} \in(a, b]$. We have the following two cases.

Case 1. $t_{0} \in(a, b)$, and $t \in\left(t_{0}, b\right]$. Then

$$
\begin{aligned}
\left|I_{a}^{\alpha} f(t)-I_{a}^{\alpha} f\left(t_{0}\right)\right| & \leq \frac{1}{\Gamma(\alpha)}\left[\int_{a}^{t_{0}}\left|(t-s)^{\alpha-1}-\left(t_{0}-s\right)^{\alpha-1}\right||f(s)| d s+\int_{t_{0}}^{t}\left|(t-s)^{\alpha-1} f(s)\right| d s\right] \\
& \leq \frac{1}{\Gamma(\alpha)}\left[\int_{a}^{t_{0}}\left|(t-s)^{\alpha-1}-\left(t_{0}-s\right)^{\alpha-1}\right||f(s)| d s+\max _{t_{0} \leq \leq \leq t}|f(s)| \int_{t_{0}}^{t}(t-s)^{\alpha-1} d s\right] \\
& =\frac{1}{\Gamma(\alpha)}\left[\int_{a}^{t_{0}}\left|(t-s)^{\alpha-1}-\left(t_{0}-s\right)^{\alpha-1}\right||f(s)| d s+\max _{t_{0} \leq s \leq t}|f(s)| \frac{\left(t-t_{0}\right)^{\alpha}}{\alpha}\right] .
\end{aligned}
$$

Clearly the right-hand side $\rightarrow 0$ as $t \rightarrow t_{0}$. This implies that $\lim _{t \rightarrow t_{0}} I_{a^{+}}^{\alpha} f(t)=I_{a^{+}}^{\alpha} f\left(t_{0}\right)$ and thus the continuity.

Case 2. $t_{0} \in(a, b]$, and $t \in\left(a, t_{0}\right)$, the proof is similar to that of case 1 .

Remark 1.

1. If $f \in C(a, b)$ and $\lim _{t \rightarrow a^{+}} f(t)=c<\infty$ then $f \in C L_{1}(a, b)$.

2. If $f \in C(a, b)$, and $\lim _{t \rightarrow a^{+}} f(t)=c<\infty$ then $(t-a)^{\sigma} f \in C[a, b]$ for all $\sigma>0$.

Lemma 14. Let $0<\sigma<\alpha<1$, and $(t-a)^{\sigma} f(t) \in C[a, b]$. Then $I_{a^{+}}^{\alpha} f$ is continuous on $[a, b]$. (This lemma is proven in [12].)

Next we extend the inequalities in [8] (Lemmas 1.1 and 4.1) to functions in $C(0, T]$.

Lemma 15. Let $f(t)$ and $g(t)$ be continuous functions in $(0, T), T>0$. Let $v(t)$ be a differentiable function for $t>0$ such that $\lim _{t \rightarrow 0^{+}} v(t)=v_{0} \leq \infty$. If 


$$
v^{\prime}(t) \leq f(t)+g(t) v(t), t \in(0, T)
$$

then,

$$
v(t) \leq v_{0} \exp \left(\int_{0}^{t} g(s) d s\right)+\int_{0}^{t} f(s) \exp \left(\int_{s}^{t} g(\tau) d \tau\right) d s, t \in(0, T) .
$$

Proof. We write (1) as

$$
\left[v^{\prime}(s)-g(s) v(s)\right] e^{\int_{s}^{t} g(\tau) d \tau} \leq f(s) e^{\int_{s}^{t} g(\tau) d \tau},
$$

and obtain

$$
\frac{d}{d s}\left(v(s) e^{\int_{s}^{t} g(\tau) d \tau}\right) \leq f(s) e^{\int_{s}^{t} g(\tau) d \tau}
$$

By integrating both sides over $(\varepsilon, t), \varepsilon>0$, we obtain

$$
v(t) \leq v(\varepsilon) e^{\int_{s}^{t} g(\tau) d \tau}+\int_{\varepsilon}^{t} f(s) e^{\int_{s}^{t} g(\tau) d \tau} d s, t \geq \varepsilon>0 .
$$

The result follows by taking the limit as $\varepsilon \rightarrow 0$.

\section{Remark 2.}

1. If $v_{0}<\infty$, and $f, g \in C L_{1}(0, T)$, then the right-hand side is bounded.

2. If $v_{0}=0$, and $g \in C L_{1}(0, T)$, then the first term of the right hand said equal to zero.

Lemma 16. Let $v(t)$ be a positive differentiable function on $(0, T)$ such that $\lim _{t \rightarrow 0^{+}} v(t)=v_{0} \geq 0$, and

$$
v^{\prime}(t) \leq h(t) v(t)+k(t) v^{p}(t), t \in(0, T)
$$

where the functions $h$ and $k$ are continuous functions on $(0, T)$, and $p \geq 0, p \neq 1$, is a constant. Then,

$$
v(t) \leq \exp \left(\int_{0}^{t} h(s) d s\right)\left[v_{0}^{q}+q \int_{0}^{t} k(s) \exp \left(-q \int_{0}^{s} h(\tau) d \tau\right) d s\right]^{\frac{1}{q}}, t \in(0, T),
$$

where $q=1-p$ and $T$ is chosen so that the expression between the brackets is positive in the interval $(0, T)$.

Proof. Let $z=\frac{v^{q}}{q}$, then $z_{0}=\lim _{t \rightarrow 0^{+}} z(t)=\frac{v_{0}^{q}}{q}$ and

$$
z^{\prime}=v^{q-1} v^{\prime} \leq v^{q-1}\left(h v+k v^{p}\right)=v^{q} h+k=q h z+k .
$$


By Lemma 15, we obtain

$$
z(t) \leq z_{0} e^{q \int_{0}^{t} h(\tau) d \tau}+\int_{0}^{t} k(s) e^{q \int_{s}^{t} h(\tau) d \tau} d s, t>0
$$

or

$$
v^{q}(t) \underset{\geq}{q \int_{0}^{t} h(\tau) d \tau}\left[v_{0}^{q}+q \int_{0}^{t} k(s) e^{-q \int_{0}^{s} h(\tau) d \tau} d s\right],
$$

where $\leq$ (respectively, $\geq$ ) hold for $q>0$ (respectively, $q<0$ ). In both cases, this estimate implies the result.

Below, we use the terms non-increasing and non-decreasing to refer to monotonic functions only.

\section{Main results}

In this section, we present and prove our main results. Without loss of generality, we take the left end of the intervals to be 0 and drop the subscript $a^{+}$.

Theorem 17. Let $a, b \in C L_{1}(0, T), T>0$, be non-negative functions, and $t^{\sigma} b(t) \in C$ $[0, T]$, where $0<\sigma<\min _{0 \leq j \leq k}\left\{\alpha-\beta_{j}\right\}<1,0 \leq \beta_{0}<\beta_{1}<\ldots<\beta_{k}<\alpha<1$. Let $c \in C[0, T]$ be a non-negative function. Let $u \in L_{1}(0, T)$ be such that $u_{1-\alpha} \in A C[0, T]$ and satisfy the inequality

$$
\left|D^{\alpha} u(t)\right| \leq a(t)+b(t) \int_{0}^{t} c(s)\left(\sum_{j=0}^{k}\left|D^{\beta_{j}} u(s)\right|\right)^{n} d s, t \in(0, T),
$$

where $n>1$ an integer.

Then,

$$
\left|D^{\alpha} u(t)\right| \leq a(t)+b(t)\left\{(L(t))^{1-n}-(n-1) \int_{0}^{t} h(s) d s\right\}^{\frac{-1}{n-1}}, t \in(0, T)
$$

provided that $g \in L_{1}(0, T)$, and

$$
[L(t)]^{n-1} \int_{0}^{t} h(s) d s<\frac{1}{n-1}
$$

where

$$
\begin{aligned}
& L(t)=\max _{0 \leq s \leq t} \int_{0}^{s} g(\tau) d \tau \\
& g(t)=2^{n-1} c(t)\left(\sum_{j=0}^{k}\left[I^{\alpha-\beta_{j}} a(t)+\frac{\mid u_{1-\alpha}(0)}{\Gamma\left(\alpha-\beta_{j}\right)} t^{\alpha-\beta_{j}-1}\right]\right)^{n},
\end{aligned}
$$


and

$$
h(t)=2^{n-1} c(t)\left(\sum_{j=0}^{k} I^{\alpha-\beta_{j}} b(t)\right)^{n}
$$

Proof. Let

$$
\phi(t)=\int_{0}^{t} c(s)\left(\sum_{j=0}^{k}\left|D^{\beta_{j}} u(s)\right|\right)^{n} d s .
$$

Then, clearly $\varphi(0)=0$,

$$
\phi^{\prime}(t)=c(t)\left(\sum_{j=0}^{k}\left|D^{\beta_{j}} u(t)\right|\right)^{n}
$$

and

$$
\left|D^{\alpha} u(t)\right| \leq a(t)+b(t) \phi(t) .
$$

By Corollary 8 and Equation 7 we have

$$
\phi^{\prime}(t) \leq c(t)\left(\sum_{j=0}^{k}\left[I^{\alpha-\beta_{j}}\left|D^{\alpha} u(t)\right|+\frac{\mid u_{1-\alpha}(0) t^{\alpha-\beta_{j}-1}}{\Gamma\left(\alpha-\beta_{j}\right)}\right]\right)^{n} .
$$

Substituting (8) into (9), and using Lemma 11, we obtain

$$
\phi^{\prime}(t) \leq 2^{n-1} c(t)\left[\left(\sum_{j=0}^{k}\left[I^{\alpha-\beta_{j}} a(t)+\frac{\mid u_{1-\alpha}(0) t^{\alpha-\beta_{j}-1}}{\Gamma\left(\alpha-\beta_{j}\right)}\right]\right)^{n}+\left(\sum_{j=0}^{k} I^{\alpha-\beta_{j}}[b(t) \phi(t)]\right)^{n}\right]
$$

Since $\varphi(t)$ is non-decreasing, we can write (10) as

$$
\phi^{\prime}(t) \leq g(t)+h(t) \phi^{n}(t),
$$

where $g(t)$ and $h(t)$ are as defined by (4) and (5).

By integrating both sides of $(11)$ over $(0, t)$ we obtain

$$
\phi(t) \leq l(t)+\int_{0}^{t} h(s) \phi^{n}(s) d s
$$

where $l(t)=\int_{0}^{t} g(s) d s$. Since $g(t)$ is non-negative and integrable, $l(t)$ is non-decreasing and continuous on $[0, T]$. Thus $\max _{0 \leq s \leq t} l(s)=L(t)$. Also from the assumptions and Lemma 14, $h(t) \in C[0 . T]$.

By applying Lemma 9 with $\omega(v)=v^{n}$ we obtain

$$
\phi(t) \leq H^{-1}\left(H(L(t))+\int_{0}^{t} h(s) d s\right), t \in[0, T],
$$


where $H(v)=\frac{v^{1-n}-v_{0}^{1-n}}{1-n}$ and $H^{-1}(x)=\left[v_{0}^{1-n}-(n-1) x\right]^{\frac{-1}{n-1}}$. That is

$$
\phi(t) \leq\left((L(t))^{1-n}-(n-1) \int_{0}^{t} h(s) d s\right)^{\frac{-1}{n-1}},
$$

as long as

$$
[L(t)]^{n-1} \int_{0}^{t} h(s) d s<\frac{1}{n-1} .
$$

Our result follows from (8) and the bound in (13).

Corollary 18. If in addition to the hypotheses of Theorem $17, u \in I^{\alpha}\left(L_{1}(0, T)\right)$ then $g$ $(t)$ reduces to

$$
g(t)=2^{n-1} c(t)\left(\sum_{j=0}^{k} a_{\alpha-\beta_{j}}(t)\right)^{n} .
$$

Proof. This follows from Theorem 5 .

Remark 3. If $\alpha-\beta_{j}>1-\frac{1}{n}$, for all $0 \leq j \leq k$, and $\left(\sum_{j=0}^{k} I^{\alpha-\beta_{j}} a(t)\right)^{n} \in L_{1}(0, T)$, then $g \in L_{1}(0, T)$.

For $n=1$ we have the following inequality

Theorem 19. Let $a, b \in C L_{1}(0, T)$ be non-negative functions. Let $c \in C(0, T]$ be a non-negative function. Let $u \in L_{1}(0, T)$ be such that $u_{1-\alpha} \in A C[0, T], 0<\alpha<1$, and satisfy the inequality

$$
\left|D^{\alpha} u(t)\right| \leq a(t)+b(t) \int_{0}^{t} c(s) \sum_{j=0}^{k}\left|D^{\beta_{j}} u(s)\right| d s, t \in(0, T)
$$

with $0 \leq \beta_{0}<\beta_{1}<\ldots<\beta_{k}<\alpha$. Then

$$
\left|D^{\alpha} u(t)\right| \leq a(t)+b(t) \int_{0}^{t} g(s) \exp \left(\int_{s}^{t} h(\tau) d \tau\right) d s, t \in(0, T)
$$

where

$$
g(t)=c(t) \sum_{j=0}^{k}\left[I^{\alpha-\beta_{j}} a(t)+\frac{\mid u_{1-\alpha}(0)}{\Gamma\left(\alpha-\beta_{j}\right)} t^{\alpha-\beta_{j}-1}\right],
$$

and

$$
h(t)=c(t) \sum_{j=0}^{k} I^{\alpha-\beta_{j}} b(t)
$$

Proof. This follows by applying Lemma 15 to (11). 
Corollary 20. If $k=0$ and $\beta_{0}=\beta$ in Theorem 19, then $g(t)$ and $h(t)$ reduce to

$$
g(t)=c(t)\left[I^{\alpha-\beta} a(t)+\frac{\left|u_{1-\alpha}(0)\right|}{\Gamma(\alpha-\beta)} t^{\alpha-\beta-1}\right],
$$

and

$$
h(t)=c(t) I^{\alpha-\beta} b(t) .
$$

Corollary 21. If in addition to the hypotheses of Theorem 19, $u \in \mathrm{I}^{\alpha}\left(L_{1}(0, T)\right)$, then $g(t)$ reduces to

$$
g(t)=c(t) \sum_{j=0}^{k} I^{\alpha-\beta_{j}} a(t)
$$

Proof. This follows from Theorem 5 .

For the next theorem we use the following expressions. Let

$$
\begin{aligned}
& L_{1}(t)=c(t)\left|u_{1-\alpha}(0)\right| \sum_{j=0}^{j} \frac{t^{\alpha-\beta_{j}-1}}{\Gamma\left(\alpha-\beta_{j}\right)}, \\
& L_{2}(t)=c(t) \sum_{j=0}^{j} I^{\alpha-\beta_{j}} a(t), \\
& L_{3}(t)=c(t) \sum_{j=0}^{j} \frac{t^{\alpha-\beta_{j}}}{\Gamma\left(\alpha-\beta_{j}+1\right)} .
\end{aligned}
$$

Theorem 22. Let $a \in C(0, T)$ be such that $\lim _{t \rightarrow 0^{+}} a(t)=a_{0}$ is non-zero and finite. Let $c \in C(0, T]$ be a non-negative function. Let $u \in L_{1}(0, T)$ be such that $u_{1-\alpha} \in A C[0, T]$, $0<\alpha<1$, and satisfy the inequality

$$
\left|D^{\alpha} u(t)\right| \leq a(t)+\int_{0}^{t} c(s)\left|D^{\alpha} u(s)\right| \sum_{j=0}^{k}\left|D^{\beta_{j}} u(s)\right| d s, t \in(0, T)
$$

where $0 \leq \beta_{0}<\beta_{1}<\ldots<\beta_{k}<\alpha$.

(a) If $a(t)$ is positive and non-decreasing then

$$
\left|D^{\alpha} u(t)\right| \leq a(t) \exp \left(\int_{0}^{t} L_{1}(s) d s\right)\left[1-\int_{0}^{t} L_{2}(s) \exp \left(\int_{0}^{s} L_{1}(\tau) d \tau\right) d s\right]^{-1}, t \in\left(0, T_{1}\right)
$$

where $T_{1}$ is the largest value of $t$ for which $\left[1-\int_{0}^{t} L_{2}(s) \exp \left(\int_{0}^{s} L_{1}(\tau) d \tau\right) d s\right]>0$.

(b) If $a(t)$ is non-negative and non-increasing then

$$
\left|D^{\alpha} u(t)\right| \leq \exp \left(\int_{0}^{t} L_{1}(s) d s\right)\left[a_{0}^{-1}-\int_{0}^{t} L_{3}(s) \exp \left(\int_{0}^{s} L_{1}(\tau) d \tau\right) d s\right]^{-1}, t \in\left(0, T_{2}\right)
$$


where $T_{2}$ is the largest value of $t$ for which

$$
\left[a_{0}^{-1}-\int_{0}^{t} L_{3}(s) \exp \left(\int_{0}^{s} L_{1}(\tau) d \tau\right) d s\right]>0 .
$$

\section{Proof.}

(a) When $a(t)$ is positive and non-decreasing we can write the inequality (19) as

$$
\frac{\left|D^{\alpha} u(t)\right|}{a(t)} \leq 1+\int_{0}^{t} \frac{c(s)}{a(s)}\left|D^{\alpha} u(s)\right| \sum_{j=0}^{k}\left|D^{\beta_{j}} u(s)\right| d s, t \in(0, T) .
$$

Let $\psi(t)$ denote the right-hand side of $(20)$. Then $\psi(0)=1$,

$$
\left|D^{\alpha} u(t)\right| \leq a(t) \psi(t),
$$

and

$$
\psi^{\prime}(t)=\frac{c(t)}{a(t)}\left|D^{\alpha} u(s)\right| \sum_{j=0}^{k}\left|D^{\beta_{j}} u(t)\right| .
$$

Since $\psi(t)$ is non-decreasing then by Corollary 8 we can write (22) in the form

$$
\psi^{\prime}(t) \leq L_{1}(t) \psi(t)+L_{2}(t) \psi^{2}(t),
$$

where $L_{1}(t)$ and $L_{2}(t)$ are as defined in (18).

Using Lemma 16 (with $p=2$ ) we obtain

$$
\psi(t) \leq \exp \left(\int_{0}^{t} L_{1}(s) d s\right)\left[1-\int_{0}^{t} L_{2}(s) \exp \left(\int_{0}^{s} L_{1}(\tau) d \tau\right) d s\right]^{-1},
$$

as long as $\left[1-\int_{0}^{t} L_{2}(s) \exp \left(\int_{0}^{s} L_{1}(\tau) d \tau\right) d s\right]>0$.

(b) When $a(t)$ is non-negative and non-increasing we can write (19) in the form

$$
\left|D^{\alpha} u(t)\right| \leq a_{0}+\int_{0}^{t} c(s)\left|D^{\alpha} u(s)\right| \sum_{j=0}^{k}\left|D^{\beta_{j}} u(s)\right| d s .
$$

Denoting the right-hand side of (23) by $\phi(t)$, we have

$$
\left|D^{\alpha} u(s)\right| \leq \varphi(t),
$$

and $\phi(t)=a_{0}$. By differentiation of $\phi$ we obtain

$$
\varphi^{\prime}(t)=c(t)\left|D^{\alpha} u(t)\right| \sum_{j=0}^{k}\left|D^{\beta_{j}} u(t)\right| \leq c(t) \varphi(t) \sum_{j=0}^{k}\left|D^{\beta_{j}} u(t)\right| .
$$

Then, we proceed as in the first part of the proof.

Corollary 23. Let $a \in C(0, T)$ be such that $\lim _{t \rightarrow 0^{+}} a(t)=a_{0}$ is non-zero and finite. Let $c \in C(0, T]$ be a non-negative function. Let $u \in I^{\alpha}\left(L_{1}(0, T)\right), 0<\alpha<1$, satisfy the inequality (19). Let 


$$
D_{1}(t)=c(t) \sum_{j=0}^{k} I^{\alpha-\beta_{j}} a(t), D_{2}(t)=c(t) \sum_{j=0}^{k} \frac{t^{\alpha-\beta_{j}}}{\Gamma\left(\alpha-\beta_{j}+1\right)} .
$$

Then,

(a) If $a(t)$ is positive and non-decreasing; and $D_{1} \in L_{1}(0, T)$, then

$$
\left|D^{\alpha} u(t)\right| \leq a(t)\left[1-\int_{0}^{t} D_{1}(s) d s\right]^{-1},
$$

as long as $\int_{0}^{t} D_{1}(s) d s<1$.

(b) If $a(t)$ is non-negative and non-increasing; and $D_{2} \in L_{1}(0, T)$, then

$$
\left|D^{\alpha} u(t)\right| \leq\left[a_{0}^{-1}-\int_{0}^{t} D_{2}(s) d s\right]^{-1}
$$

as long as $\left[a_{0}^{-1}-\int_{0}^{t} D_{2}(s) d s\right]>0$.

Proof. The result follows from Theorem 5 and Corollary 8 .

For the next theorem, we introduce the following expressions.

$$
\begin{aligned}
& K_{1}(t)=\frac{c(t) u_{1-\alpha}^{2}(0)}{a(t)} \sum_{i=0}^{m} \sum_{j=0}^{k} \frac{t^{2 \alpha-\beta_{j}-\gamma_{i}-2}}{\Gamma\left(\alpha-\beta_{j}\right) \Gamma\left(\alpha-\gamma_{i}\right)} \\
& K_{2}(t)=\frac{c(t)\left|u_{1-\alpha}(0)\right|}{a(t)} \sum_{i=0}^{m} \sum_{j=0}^{k}\left(\frac{t^{\alpha-\beta_{j}-1}}{\Gamma\left(\alpha-\beta_{j}\right)} I^{\alpha-\gamma_{i}} a(t)+\frac{t^{\alpha-\gamma_{i}-1}}{\Gamma\left(\alpha-\gamma_{i}\right)} I^{\alpha-\beta_{j}} a(t)\right), \\
& K_{3}(t)=\frac{c(t)}{a(t)} \sum_{i=0}^{m} \sum_{j=0}^{k} I^{\alpha-\beta_{j}} a(t) I^{\alpha-\gamma_{i}} a(t), \\
& \bar{K}(t)=\max _{0 \leq s \leq t}\left[1+\int_{0}^{s} K_{1}(\tau) d \tau\right] \\
& \bar{K}_{0}(t)=\max _{0 \leq s \leq t}\left[a_{0}+\int_{0}^{s} a(\tau) K_{1}(\tau) d \tau\right] .
\end{aligned}
$$

Theorem 24. Let $a \in C(0, T)$ with $\lim _{t \rightarrow 0^{+}} a(t)=a_{0}$ non-zero and finite. Let $c \in C[0, T]$ be non-negative. Let $u \in L_{1}(0, T)$ be such that $u_{1-\alpha} \in A C[0, T], 0<\alpha<1$, and satisfy the inequality

$$
\left|D^{\alpha} u(t)\right| \leq a(t)+\int_{0}^{t} c(s) \sum_{i=0}^{m}\left|D^{\gamma_{i}} u(s)\right| \sum_{j=0}^{k}\left|D^{\beta_{j}} u(s)\right| d s, t \in(0, T),
$$

where $0 \leq \beta_{0}<\beta_{1}<\ldots<\beta_{k}<\alpha, 0 \leq \gamma_{0}<\gamma_{1}<\ldots<\gamma_{m}<\alpha$. 
(a) If $a(t)$ is positive and non-decreasing; $K_{1} \in L_{1}(0, T)$, and $K_{2}, K_{3} \in C[0, T)$, then

$$
\left|D^{\alpha} u(t)\right| \leq a(t) \bar{K}(t) \exp \left(\int_{0}^{t} K_{2}(s) d s\right)\left[1-\bar{K}(t) \exp \left(\int_{0}^{t} K_{2}(s) d s\right) \int_{0}^{t} K_{3}(s) d s\right]^{-1}, t \in\left(0, T_{3}\right),
$$

where $T_{3}$ is the largest value of $t$ for which the bracket is positive.

(b) If $a(t)$ is non-negative and non-increasing; $a(t) K_{1}(t) \in L_{1}(0, T), a(t) K_{2}(t), a(t) K_{3}$ $(t) \in C[0, T)$, then

$$
\left|D^{\alpha} u(t)\right| \leq \bar{K}_{0}(t) \exp \left(\int_{0}^{t} a(s) K_{2}(s) d s\right)\left[1-\bar{K}_{0}(t) \exp \left(\int_{0}^{t} a(s) K_{2}(s) d s\right) \int_{0}^{t} a(s) K_{3}(s) d s\right]^{-1},
$$

$t \in\left(0, T_{4}\right)$, where $T_{4}$ is the largest value of $t$ for which the bracket is positive.

\section{Proof.}

(a) Suppose $a(t)$ is positive and non-decreasing. Then, we can write the inequality (25) as

$$
\frac{\left|D^{\alpha} u(t)\right|}{a(t)} \leq 1+\int_{0}^{t} \frac{c(s)}{a(s)} \sum_{i=0}^{m}\left|D^{\gamma_{i}} u(s)\right| \sum_{j=0}^{k}\left|D^{\beta_{j}} u(s)\right| d s .
$$

Let $\psi(t)$ denote the right-hand side of $(26)$. Then, $\psi(0)=1$,

$$
\left|D^{\alpha} u(t)\right| \leq a(t) \psi(t),
$$

and

$$
\psi^{\prime}(t)=\frac{c(t)}{a(t)} \sum_{i=0}^{m}\left|D^{\gamma_{i}} u(t)\right| \sum_{j=0}^{k}\left|D^{\beta_{j}} u(t)\right| .
$$

Since $\psi$ is non-decreasing, by Corollary 8 we have

$$
\left|D^{\beta_{j}} u(t)\right| \leq \psi(t) I^{\alpha-\beta_{j}} a(t)+\frac{\left|u_{1-\alpha}(0)\right| t^{\alpha-\beta_{j}-1}}{\Gamma\left(\alpha-\beta_{j}\right)}, j=0,1,2, \ldots, k .
$$

and

$$
\left|D^{\gamma_{i}} u(t)\right| \leq \psi(t) I^{\alpha-\gamma_{i}} a(t)+\frac{\left|u_{1-\alpha}(0)\right| t^{\alpha-\gamma_{i}-1}}{\Gamma\left(\alpha-\gamma_{i}\right)}, i=0,1,2, \ldots, m
$$

By substituting (29) and (30) into (28) and since $\psi$ is non-decreasing, we obtain

$$
\psi^{\prime} \leq K_{1}(t)+K_{2}(t) \psi(t)+K_{3}(t) \psi^{2}(t),
$$

where $K_{1}(t), K_{2}(t)$ and $K_{3}(t)$ are as defined in (24). By integrating (31) we obtain

$$
\psi(t) \leq 1+\int_{0}^{t} K_{1}(s) d s+\int_{0}^{t} K_{2}(s) \psi(s) d s+\int_{0}^{t} K_{3}(s) \psi^{2}(s) d s .
$$


Applying Lemma 10 with

$$
\begin{aligned}
& c_{0}(t)=\max _{0 \leq s \leq t}\left[1+\int_{0}^{s} K_{1}(\tau) d \tau\right], \\
& c_{1}(t)=c_{0}(t) \exp \int_{0}^{t} K_{2}(s) d s, \\
& c_{2}(t)=\left[c_{1}^{-1}(t)-\int_{0}^{t} K_{3}(s) d s\right]^{-1} .
\end{aligned}
$$

We obtain our result.

(b) Suppose $a(t)$ is non-negative and non-increasing. Then, we can write (25) in the form

$$
\left|D^{\alpha} u(t)\right| \leq a_{0}+\int_{0}^{t} c(s) \sum_{i=1}^{m}\left|D^{\gamma_{i}} u(s)\right| \sum_{j=1}^{k}\left|D^{\beta_{j}} u(s)\right| d s .
$$

Denoting the right-hand side of (33) by $\phi(t)$, we have $\left|D^{\alpha} u(t)\right| \leq \phi(t), \phi(0)=a_{0}$, and

$$
\varphi^{\prime}(t)=c(t) \sum_{i=1}^{m}\left|D^{\gamma_{i}} u(t)\right| \sum_{j=1}^{k}\left|D^{\beta_{j}} u(t)\right| .
$$

The reset of the proof is similar to that of the first part.

Corollary 25. Let $a \in C(0, T)$, with $\lim _{t \rightarrow 0^{+}} a(t)=a_{0}$ non-zero and finite. Let $c \in C[0$, $T]$ be non-negative. Let $u \in I^{\alpha}\left(L_{1}(0, T)\right), 0<\alpha<1$, satisfy the inequality (25). Let

$$
\begin{aligned}
& K_{1}(t)=\frac{c(t)}{a(t)} \sum_{i=0}^{m} \sum_{j=0}^{k} I^{\alpha-\gamma_{i}} a(t) I^{\alpha-\beta_{j}} a(t), \\
& K_{2}(t)=c(t) \sum_{j=0}^{k} \sum_{i=0}^{m} \frac{t^{2 \alpha-\beta_{j}-\gamma_{i}}}{\Gamma\left(\alpha-\beta_{j}+1\right) \Gamma\left(\alpha-\gamma_{i}+1\right)} .
\end{aligned}
$$

(a) If $a(t)$ is positive and non-decreasing; and if $K_{1} \in L_{1}(0, T)$, then

$$
\left|D^{\alpha} u(t)\right| \leq a(t)\left[1-\int_{0}^{t} K_{1}(s) d s\right]^{-1},
$$

as long as $\int_{0}^{t} K_{1}(s) d s<1$.

(b) If $a(t)$ is non-negative and non-increasing and $K_{2} \in L_{1}(0, T)$, then

$$
\left|D^{\alpha} u(t)\right| \leq\left[a_{0}^{-1}-\int_{0}^{t} K_{2}(s) d s\right]^{-1},
$$

as long as $\left[a_{0}^{-1}-\int_{0}^{t} K_{2}(s) d s\right]>0$. 
Proof. The result follows from Theorem 5 and Corollary 8 .

\section{Applications}

In this section, we illustrate our previous results by some applications. In particular, we show how to use these results to prove existence and determine the asymptotic behavior for some classes of fractional differential equations.

We consider the following Cauchy-type problem

$$
\begin{aligned}
& D^{\alpha} u(t)=f\left(t, D^{\beta_{0}} u(t), D^{\beta_{1}} u(t), \ldots D^{\beta_{k}} u(t)\right), t>0, \\
& I^{1-\alpha} u(0)=u_{0} \in R
\end{aligned}
$$

where $0 \leq \beta_{0}<\beta_{1}<\ldots<\beta_{k}<\alpha<1$, and $f$ is a continuous function in all its variables.

Proposition 26. If $u(t)$ has a assumable fractional derivative $D^{\beta} u$ in $(0, T), 0 \leq \beta \leq 1$, then for $\alpha \geq \beta$,

$$
I^{\alpha} D^{\beta} u(t)=u_{\alpha-\beta}(t)-\frac{u_{1-\beta}(0)}{\Gamma(\alpha)} t^{\alpha-1} .
$$

(See [[11], p. 48].) In particular, we have

Theorem 27. If $u \in L_{1}(0, T)$ such that $I^{1-\alpha} u \in A C[0, T]$ and satisfy the problem (34), then

$$
u(t)=I^{\alpha} f\left(t,\left\{D^{\beta_{j}} u(t)\right\}_{j=0}^{k}\right)+\frac{u_{0}}{\Gamma(\alpha)} t^{\alpha-1} .
$$

Theorem 28. Suppose

$$
\left|f\left(t, D^{\beta_{0}} u, D^{\beta_{1}} u, \ldots, D^{\beta_{k}} u\right)\right| \leq a(t)+b(t) \int_{0}^{t} c(s)\left(\sum_{j=0}^{k} \mid D^{\beta_{j}} u(s)\right)^{n} d s, t>0,
$$

with $a, b \in C L_{1}(0, T)$ and $c \in C[0, T]$ are non-negative, and $t^{\sigma} b(t) \in C[0, T]$, $0<\sigma<\min _{0 \leq j \leq k}\left\{\alpha-\beta_{j}\right\}<1$. Further suppose the following hold.

(a) $\alpha-\beta_{j}>1-\frac{1}{n}$, for all $0 \leq j \leq k$,

(b) $\left(\sum_{j=0}^{k} I^{\alpha-\beta_{j}} a(t)\right)^{n} \in L_{1}(0, T)$,

(c) $(L(t))^{n-1} \int_{0}^{t} h(s) d s<\frac{1}{n-1}, L(t)=\max _{0 \leq s \leq t} \int_{0}^{s} g(\tau) d \tau$, where $g(t)$ and $h(t)$ are as defined by (4) and (5).

If $u \in L_{1}(0, T)$ is a local solution of (34) that has a summable fractional derivative $D^{\alpha}$ $u(t)$, then this solution exists for $t \in\left(0, T_{0}\right)$, where $T_{0}$ is the largest value in $(0, T)$ such that $(L(t))^{n-1} \int_{0}^{t} h(s) d s<\frac{1}{n-1}$.

Proof. Following the proof of Theorem 17, we have

$$
\left|D^{\alpha} u(t)\right| \leq a(t)+b(t)\left\{(L(t))^{1-n}-(n-1) \int_{0}^{t} h(s) d s\right\}^{\frac{-1}{n-1}}=B(t)
$$


for all $0<t<T_{0}$. By theorem 27, we have

$$
|u(t)| \leq\left|I^{\alpha} D^{\alpha} u\right|+\frac{\left|u_{0}\right|}{\Gamma(\alpha)} t^{\alpha-1} \leq I^{\alpha} B(t)+\frac{\left|u_{0}\right|}{\Gamma(\alpha)} t^{\alpha-1} .
$$

Therefore, $u(t)$ is bounded by a continuous function.

Example 1. Consider the problem

$$
\begin{aligned}
& D^{0.8} u(t)=f(t, u(t)), t \in(0, \pi], \\
& I^{0.2} u(0)=1,
\end{aligned}
$$

with $f$ satisfying the inequality

$$
|f(t, u(t))| \leq \frac{\sin t}{\sqrt{t}}+\frac{1}{\sqrt{t}} \int_{0}^{t} s^{2} u^{2}(s) d s \leq \frac{1}{\sqrt{t}}+\frac{1}{\sqrt{t}} \int_{0}^{t} s^{2} u^{2}(s) d s .
$$

Then, we can take $T_{0}=0.17826$. Thus, if $u \in L_{1}(0, \pi)$ is a local solution with a summable fractional derivative $D^{0.8} u(t)$, this solution exists for $0<t<0.17826$.

Theorem 29. Suppose that

$$
\left|f\left(t, D^{\beta_{0}} u, D^{\beta_{1}} u, \ldots, D^{\beta_{k}} u\right)\right| \leq a(t)+\int_{0}^{t} c(s)\left|D^{\alpha} u(s)\right| \sum_{j=0}^{k}\left|D^{\beta_{j}} u(s)\right| d s, t>0,
$$

where $0 \leq \beta_{0}<\beta_{1}<\ldots<\beta_{k}<\alpha<1, a \in C(0, T)$ with $\lim _{t \rightarrow 0^{+}} a(t)=a_{0}$ non-zero and finite, and $c \in C(0, T)$ is non-negative. Let $L_{k}(t), k=1,2,3$, and $T_{j}, j=1,2$ be as in Theorem 22.

Then, we consider the following cases.

(a) $a(t)$ is positive and non-decreasing, $L_{1}, L_{2} \in C L_{1}(0, T)$, and

$$
1-\int_{0}^{t} L_{2}(s) \exp \left(\int_{0}^{s} L_{1}(\tau) d \tau\right) d s>0, t \in\left(0, T_{1}\right)
$$

In this case, if $u \in L_{1}(0, T)$ is a local solution of (34) that has a summable fractional derivative $D^{\alpha} u(t)$, then this solution exists for all $t \in\left(0, T_{1}\right)$.

(b) $a(t)$ is non-negative and non-increasing, $L_{1}, L_{3} \in C L_{1}(0, T)$, and

$$
a_{0}^{-1}-\int_{0}^{t} L_{3}(s) \exp \left(\int_{0}^{s} L_{1}(\tau) d \tau\right) d s>0, t \in\left(0, T_{2}\right)
$$

In this case, if $u \in L_{1}(0, T)$ is a local solution of (34) that has a summable fractional derivative $D^{\alpha} u(t)$, then this solution exists for all $t \in\left(0, T_{2}\right)$

Proof. The result follows from Theorems 22 and 27.

Example 2. Consider the problem

$$
\begin{aligned}
& D^{0.7} u(t)=f\left(t, D^{0.2} u(t)\right), t \in(0,2], \\
& I^{0.3} u(0)=0.1
\end{aligned}
$$


with $f$ satisfying the inequality

$$
\left|f\left(t, D^{0.2} u(t)\right)\right| \leq \frac{t}{e^{t}-1}+\int_{0}^{t} \frac{1}{\sqrt[3]{s}}\left|D^{0.7} u(s)\right|\left|D^{0.2} u(s)\right| d s .
$$

We have $L_{1}(t)=0, L_{3}(t)=1.14 t^{0.17} \in C L_{1}(0,1)$, and 1-0.97 $t^{0.17}>0, t \in(0,1.02638)$. Thus, if $u \in L_{1}(0,2)$ is a local solution with a summable fractional derivative $D^{0.7} u$ $(t)$, then this solution exists for all $t \in(0,1.02638)$.

Theorem 30. Suppose that

$$
\left|f\left(t, u, D^{\beta_{0}} u, \ldots, D^{\beta_{k}} u\right)\right| \leq a(t)+\int_{0}^{t} c(s) \sum_{i=0}^{m}\left|D^{\gamma_{i}} u(s)\right| \sum_{j=0}^{k}\left|D^{\beta_{j}} u(s)\right| d s, t \in(0, T),
$$

where $0 \leq \beta_{0}<\beta_{1}<\ldots<\beta_{k}<\alpha<1,0 \leq \gamma_{0}<\gamma_{1}<\ldots<\gamma_{m}<\alpha<1, a \in C(0, T)$ with $\lim _{t \rightarrow 0^{+}} a(t)=a_{0}$ non-zero and finite, and $c \in C[0, T]$ is non-negative. Let $K_{i}(t), i=1,2$, $3, \bar{K}(t), \bar{K}_{0}(t), T_{3}$ and $T_{4}$ be as in Theorem 24 . Then, we consider the following cases.

(a) $a(t)$ is positive and non-decreasing, $\frac{c(t)}{a(t)} t^{2 \alpha-\beta_{j}-\gamma_{i}-2} \in L_{1}(0, T)$ for all $i, j, K_{2}, K_{3}$ $\in C[0, T)$, and

$$
1-\bar{K}(t) \exp \left(\int_{0}^{t} K_{2}(s) d s\right) \int_{0}^{t} K_{3}(s) d s>0, t \in\left(0, T_{3}\right) .
$$

In this case, if $u \in L_{1}(0, T)$ is a local solution of (34) that has a summable fractional derivative $D^{\alpha} u(t)$, then this solution exists for all $t \in\left(0, T_{3}\right)$.

(b) $a(t)$ is non-negative and non-increasing, a $(t) K_{2}(t), a(t) K_{3}(t) \in C[0, T)$, $c(t) t^{2 \alpha-\beta_{j}-\gamma_{i}-2} \in L_{1}(0, T)$ for all $i, j$, and

$$
1-\bar{K}_{0}(t) \exp \left(\int_{0}^{t} a(s) K_{2}(s) d s\right) \int_{0}^{t} a(\tau) K_{3}(s) d s>0, t \in\left(0, T_{4}\right) .
$$

In this case, if $u \in L_{1}(0, T)$ is a local solution of (34) that has a summable fractional derivative $D^{\alpha} u(t)$, then this solution exists for all $t \in\left(0, T_{4}\right)$.

Proof. The result follows from Theorems 24 and 27.

Example 3. Consider the problem

$$
\begin{aligned}
& D^{0.6} u(t)=f(t, u(t)), t \in(0,1], \\
& I^{0.4} u(0)=0.5,
\end{aligned}
$$

with $f$ satisfying the inequality

$$
|f(t, u(t))| \leq \frac{e^{t}-1}{\sqrt{t}}+\int_{0}^{t}\left(e^{s}-1\right) u^{2}(s) d s .
$$


By calculation we have $1-3.26 t^{1.7} e^{1.05 t^{1.2}}>0$ for $t \in(0,0.404986)$. Thus, if $u \in L_{1}$ $(0,1)$ is a local solution with a summable fractional derivative $D^{0.6} u(t)$, then this solution exists for all $t \in(0,0.404986)$.

Example 4. Consider the problem

$$
\begin{aligned}
& D^{0.7} u(t)=f(t, u(t)), t \in(0,1], \\
& I^{0.3} u(0)=0.1,
\end{aligned}
$$

with $f$ satisfy the inequality

$$
|f(t, u(t))| \leq \frac{\sin \sqrt{t}}{2 \sqrt{t}}+\int_{0}^{t}\left(e^{s}-1\right) u^{2}(s) d s
$$

It follows that if $u \in L_{1}(0,1)$ is a local solution with a summable fractional derivative $D^{0.7} u(t)$, then this solution exists for all $t \in(0,1)$.

Finally, we show how the results in Section 3 can provide information about the behavior of the solutions for large values of $t$. For this purpose, we utilize the following lemma which is proved in [13].

Lemma. 31. Let $\alpha, \lambda, \omega>0$, then

$$
t^{1-\alpha} \int_{0}^{t}(t-s)^{\alpha-1} s^{\lambda-1} e^{-\omega s} d s \leq \text { Const. } t>0
$$

Theorem 32. Suppose $f$ in (34) satisfy the inequality

$$
\left|f\left(t, D^{\beta_{0}} u, D^{\beta_{1}} u, \ldots D^{\beta_{k}} u\right)\right| \leq a(t)+b(t) \int_{0}^{t} c(s) \sum_{j=0}^{k}\left|D^{\beta_{j}} u(s)\right| d s, t>0,
$$

with $0 \leq \beta_{0}<\beta_{1}<\ldots<\beta_{k}<\alpha<1, a, b \in C L_{1}(0, T)$ and $c \in C(0, T]$ are non-negative. Further, suppose the following.

1. $\int_{0}^{t} g(s) \exp \left(\int_{s}^{t} h(\tau) d \tau\right) d s \leq M$, where $M$ is a positive constant, and $g(t), h(t)$ are as defined by (16) and (17).

2. $a(t) \leq M_{1} t^{\lambda_{1}-1} e^{-\omega_{1} t}, M_{1}, \lambda_{1}, \omega_{1}>0$.

3. $b(t) \leq M_{2} t^{\lambda_{2}-1} e^{-\omega_{2} t}, M_{2}, \lambda_{2}, \omega_{2}>0$.

If $u \in L_{1}(0, T)$ is a local solution of (34) that has a summable fractional derivative $D^{\alpha}$ $u(t)$, then

$$
|u(t)| \leq \frac{C}{t^{1-\alpha}}, t>0,
$$

where $C$ is positive constant.

Proof. From Theorems 27 and 19, we have

$$
|u(t)| \leq I^{\alpha} Z(t)+\frac{\left|u_{0}\right|}{\Gamma(\alpha)} t^{\alpha-1},
$$


where

$$
Z(t)=a(t)+b(t) \int_{0}^{t} g(s) \exp \left(\int_{s}^{t} h(\tau) d \tau\right) d s .
$$

Since $\int_{0}^{t} g(s) \exp \left(\int_{s}^{t} h(\tau) d \tau\right) d s \leq M$, then

$$
Z(t) \leq a(t)+M b(t)
$$

and

$$
t^{1-\alpha}|u(t)| \leq t^{1-\alpha} I^{\alpha} a(t)+M t^{1-\alpha} I^{\alpha} b(t)+\frac{\left|u_{0}\right|}{\Gamma(\alpha)} .
$$

The conditions 2-3 and Lemma 31 yield the result.

\section{Acknowledgements}

The authors are very grateful for the financial support provided by the King Fahd University of Petroleum and Minerals and Princess Nora Bint Abdurrahman University.

\section{Author details}

${ }^{1}$ Department of Mathematical Sciences, Princess Nora Bint Abdulrahman University, Riyadh 84428, Saudi Arabia

${ }^{2}$ Department of Mathematics \& Statistics, King Fahd University of Petroleum \& Minerals, Dhahran 31261, Saudi Arabia

\section{Authors' contributions}

Both authors worked jointly on drafting and approving the final manuscript.

\section{Competing interests}

The authors declare that they have no competing interests.

Received: 2 July 2011 Accepted: 10 November 2011 Published: 10 November 2011

\section{References}

1. Kilbas, AA, Srivastava, HM, Trujillo, Jj: Theory and Applications of Fractional Differential Equations. In Mathematics Studies, vol. 204,Elsevier (2006)

2. Baleanu, D, Mustafa, O, Agarwal, R: Asymptotically linear solutions for some linear fractional differential equations. Abstr Appl Anal 8 (2010). (Article ID 865139)

3. Baleanu, D, Mustafa, O, Agarwal, R: An existence result for a superlinear fractional differential equation. Appl Math Lett. 23(9), 1129-1132 (2010). doi:10.1016/.j.aml.2010.04.049

4. Anastassiou, GA: Fractional Differentiation Inequalities. Springer (2009)

5. Furati, KM, Tatar, N-E: Some fractional differential inequalities and their applications. Math Inequal Appl. 9(4), 577-598 (2006)

6. Furati, KM, Tatar, N-E: Inequalities for fractional differential equations. Math Inequal Appl. 12(2), 279-293 (2009)

7. Furati, KM: A fractional differential inequality with application. Nonlinear Anal Theory Methods Appl. 74(13), 4330-4337 (2011). doi:10.1016/j.na.2011.03.027

8. Bainov, D, Simeonov, P: Integral Inequalities and Applications. Kluwer Academic Publishers57 (1992)

9. Pachpatte, BG: Inequalities for differential and integral equations. In: Ames WF (ed.) Mathematics in Science and Engineering, vol. 197, Academic Press (1998)

10. Kolomogorov, AN, Fomin, SV: Fundamentals of the Theory of Functions and Functional Analysis (Russian). Moscow. (1986)

11. Samko, SG, Kilbas, AA, Marichev, OI: Fractional Integral Derivatives, Theory and Applications. Gorden and Breach, Amsterdam (1993)

12. Bai, C, Fang, J: The existence of a positive solution for singular coupled system of nonlinear fractional differential equations. Appl Math Comput. 150, 611-621 (2004). doi:10.1016/S0096-3003(03)00294-7

13. Kirane, M, Tatar, N-e: Global existence and stability of some semilinear problems. Arch Math. 36(1), 33-44 (2000)

doi:10.1186/1029-242X-2011-110

Cite this article as: Al-Jaser and Furati: Singular fractional integro-differential inequalities and applications. Journal of Inequalities and Applications 2011 2011:110. 